11,01

\title{
Неравновесные диффузионные фазовые превращения в сплавах, обусловленные миграцией границ зерен и дислокаций
}

\author{
(C) И.К. Разумов ${ }^{1}$, Ю.Н. Горностырев ${ }^{1,2}$, А.Е. Ермаков ${ }^{1,2}$ \\ ${ }^{1}$ Институт фризики металлов им. М.Н. Михеева УрО РАН, \\ Екатеринбург, Россия \\ ${ }^{2}$ Уральский федеральный университет им. Б.Н. Ельцина, \\ Екатеринбург, Россия \\ E-mail: rik@imp.uran.ru
}

(Поступила в Редакцию 25 сентября 2018 г.)

Рассмотрены основные сценарии неравновесных диффузионных превращений, обусловленных движущимися дефектами (дислокации, границы зерен) при интенсивной пластической деформации сплавов. Показано, что изменение фазового состояния реализуется локально в области дефекта, в измененных термодинамических условиях, и достигнутое состояние замораживается после смещения дефекта в силу различия скоростей объемной диффузии и диффузии на дефекте. Поэтому в процессе воздействия сплав удаляется от состояния термодинамического равновесия, так что в зависимости от типа системы, возможны различные изменения структурно-фазового состояния, такие как разупорядочение сплава, растворение выделений равновесных фаз, появление неравновесных фаз, формирование регулярных структур. Эти эффекты могут иметь место, если обработка сплава проводится при умеренных температурах, когда объемная диффузия заморожена, а на дефектах - достаточно активна. В рамках сформулированных представлений обсуждаются явления фазовой и структурной неустойчивости, развивающиеся в условиях интенсивной пластической деформации при умеренных температурах.

Работа выполнена в рамках государственного задания по темам „Магнит“ N AAAA-A18-118020290129-5 и „Структура“ N AАAА-A18-118020190116-6.

DOI: 10.21883/FTT.2019.02.47137.257

\section{1. Введение}

В последние десятилетия значительное внимание привлекают необычные фазовые и структурные превращения, которые реализуются в сплавах в процессе интенсивной пластической деформации (ИПД) или последующей термической обработки. К ним относятся, в частности, разупорядочение и аморфизация сплавов [1,2], формирование пересышенных твердых растворов несмешиваемых компонент [3,4], распад с выделением неравновесных фаз [5,6], циклические реакции [7], формирование модулированных структур (patterns), устойчивых в ходе продолжающегося воздействия $[8,9]$, аномально быстрая реализация низкотемпературных фаз $[10,11]$ и широких зернограничных сегрегаций [12-14].

Процессы разупорядочения или аномального механосплавления в принципе могут быть поняты в терминах прямого перемешивания атомов, обеспечиваемого развитием полос скольжения в пересекающихся плоскостях [15-17]. С другой стороны, наблюдаемое ускорение аномального механосплавления при переходе к нанокристаллическому состоянию $[18,19]$, когда проникновение дислокаций в объем зерна затруднено [20] не может быть понято в рамках механизма, предложенного в [15-17]. Кажется несомненным, что при умеренных температурах, наряду с процессами перемешивания необходимо учитывать также процессы диффузионного массопереноса. В частности, явления распада и реализация низ- котемпературных фаз в условиях ИПД при комнатной температуре свидетельствуют о протекании аномально быстрой диффузии. Оценки показывают, что в этом случае превращения реализуются, вероятно, за счет диффузии по дислокациям и границам зерен с участием неравновесных точечных дефектов [10,13,21,22], генерируемых при ИПД.

Диффузия на дислокациях и границах зерен (ГЗ) не только обеспечивает ускоренное развитие равновесных фазовых превращений, но и приводит к реализации неравновесных фазовых и структурных состояний. В частности, эффект „диффузионного перерезания“ выделений дислокациями хорошо известен [23-25], и считается одной из основных причин растворения частиц интерметаллидов и карбидных выделений в сталях при ИПД [4]. Отметим, что в работах [23-25] учитывалось только упругое взаимодействие атомов с дислокациями. Однако, в настоящее время не вызывает сомнения, что упругий вклад является далеко не единственным, определяющим взаимодействие растворенных атомов с другими дефектами решетки [26]. Более существенную роль может играть изменение энергий химической связи при перемещении атомов из объема выделения в область дефекта (см., например, [26,27]), что необходимо учитывать при построении последовательной модели.

Модель „диффузионного перерезания“ [23-25] рассматривает эволюцию формы выделения в поле напряжений, создаваемых ансамблем краевых дислокаций 
на межфазной границе. Между тем, в условиях ИПД движущая сила миграции дефектов достаточно велика, и может приводить к механическому перерезанию дислокациями (полу)когерентных выделений [28,29]. Многократное перерезание выделений дислокациями приводит к их измельчению и перемешиванию сортов атомов [15-17]. При этом объемная диффузия не всегда выступает конкурирующим фактором, и в определенных случаях может даже способствовать растворению выделений [30]. Однако модели [15-17] не принимают во внимание диффузионные процессы, развивающиеся на дислокациях при умеренных температурах. Так в работе [31] было показано, что изменение термодинамических свойств сплава (энергии сегрегации, смешения и упорядочения) в ядрах дислокаций, перерезающих выделение, может стимулировать локальное развитие неравновесного превращения. При смещении дислокации достигнутое состояние замораживается, так что по мере развития деформации состояние сплава удалялся от термодинамического равновесия.

Эффект растворения выделений движущимися границами зерен, впервые обнаруженный в работе [32], обычно наблюдается при повышенных температурах, в условиях собирательной рекристаллизации [33-35]. Можно ожидать, что в случае ИПД аналогичные процессы развиваются в условиях низкотемпературной динамической рекристаллизации [36]. Основываясь на результатах расчетов $[37,38]$ профиля концентрации примеси вблизи движущейся ГЗ, в работе [35] было показано, что мелкие выделения растворяются в обедненной зоне вблизи ГЗ, при условии, что их размер сопоставим с шириной этой зоны, а скорость движения границы не слишком высока. В литературе отсутствуют модели, рассматривающие прохождение ГЗ через выделение другой фазы. Между тем, можно ожидать, что в условиях ИПД, когда движущая сила миграции ГЗ достаточно велика, этот процесс будет приводить к существенному изменению фазового состояния сплава.

При анализе фазовых превращений обусловленных миграцией ГЗ, необходимо прежде всего рассмотреть сегрегации на движущихся ГЗ, чему были посвящены работы [37-42]. В работах [37,38] внимание фокусировалось на стационарном режиме, и был сделан вывод, что сегрегация на движущейся ГЗ выражена слабее, чем на неподвижной. Однако, при умеренных температурах в разбавленных сплавах важна стадия формирования сегрегаций, в то время как стационарное состояние может быть не достигнуто за разумные времена. В работах $[41,42]$ было проведено моделирование кинетики формирования сегрегаций и показано, в частности, что при некоторых специальных режимах движения достигаемая концентрация примеси на ГЗ может быть выше, чем в стационарном режиме.

Таким образом, можно ожидать, что движущиеся дислокации и ГЗ могут оказывать существенное влияние на развитие фазовых превращений в условиях ИПД. В настоящей работе мы показываем, что при умеренных температурах, в предположении действия достаточных движущих сил, обеспечивающих миграцию протяженных дефектов, диффузия на них может приводить к целому спектру неравновесных превращений, включая быстрое разупорядочение, реализацию неравновесных фаз, формирование пересыщенных твердых растворов и диссипативных структур. При этом механическая энергия преобразуется во внутреннюю энергию сплава, и он удаляется от состояния термодинамического равновесия.

\section{2. Формулировка модели}

В приближении среднего поля плотность свободной энергии сплава $\mathrm{AB}$ с упорядочением может быть записана в виде [43-45]

$$
\begin{aligned}
f(c, \mathbf{r}) & =\varepsilon(\mathbf{r}) c+v(\mathbf{r}) c^{2}+\theta(\mathbf{r}) \eta^{2} \\
+ & \frac{k T}{2} \sum_{n}\left[c^{(n)} \ln c^{(n)}+\left(1-c^{(n)}\right) \ln \left(1-c^{(n)}\right)\right],
\end{aligned}
$$

где $\varepsilon(\mathbf{r}), v(\mathbf{r}), \theta(\mathbf{r})$ - энергии растворения, смешения и упорядочения; $c, \eta$ - локальные концентрация выбранного компонента и степень упорядочения, связанные с подрешеточными концентрациями, $c=\left(c^{(1)}+c^{(2)}\right) / 2$, $\eta=\left(c^{(1)}-c^{(2)}\right) / 2$; суммирование проводится по двум подрешеткам $(n=1,2)$. Заметим, что запись свободной энергии в форме (1) справедлива для сплавов, упорядочение которых описывается одним семейством концентрационных волн (один параметр порядка) [43].

Мы рассматриваем структурно неоднородный сплав полагая, что параметры $\varepsilon(\mathbf{r}), v(\mathbf{r}), \theta(\mathbf{r})$ могут зависеть от координат; в частности, они локально изменяются в области протяженных структурных дефектов (дислокаций и границ зерен). В отсутствие распада и упорядочения неоднородность в распределении компонента $B$ обусловлена изменением энергии растворения

$$
\varepsilon(\mathbf{r})=\varepsilon_{b}+\delta \varepsilon \Omega\left(\mathbf{r}-\mathbf{r}_{\text {def }}\right),
$$

где $\delta \varepsilon=\varepsilon_{\mathrm{def}}-\varepsilon_{b}-$ энергия сегрегации примеси на дефекте, $\Omega\left(\mathbf{r}-\mathbf{r}_{\mathrm{def}}\right)$ - функция формы возмущения вблизи дефекта, отличная от нуля в узкой области ширины $d$, и быстро убывающая до нуля с ростом расстояния (см. Приложение). Изменение энергий смешения и упорядочения $v(\mathbf{r}), \theta(\mathbf{r})$ в области дефекта определим аналогичным образом

$$
\begin{gathered}
v(\mathbf{r})=v_{b}+\delta \nu \Omega\left(\mathbf{r}-\mathbf{r}_{\mathrm{def}}\right), \\
\theta(\mathbf{r})=\theta_{b}+\delta \theta \Omega\left(\mathbf{r}-\mathbf{r}_{\mathrm{def}}\right) .
\end{gathered}
$$

Таким образом, структурный дефект моделируется в виде узкой области, в пределах которой свойства сплава отличаются от объемных. Будем полагать, что дефект движется со скоростью $V_{\text {def, }}$ так что его положение дается радиус-вектором

$$
\mathbf{r}_{\mathrm{GB}}(t)=\mathbf{r}_{\mathrm{GB}}^{(0)}-\mathbf{V}_{\mathrm{def}} t .
$$


При таком упрощенном подходе дислокации и ГЗ отличаются только размерностью дефекта и параметрами, описывающими его взаимодействие с примесными атомами. Это позволяет рассматривать взаимодействие дислокаций и ГЗ с выделениями в рамках одного подхода. Далее мы рассматриваем в основном миграцию ГЗ, ограничиваясь обсуждением применимости полученных результатов к дислокациям.

Эволюция распределения компонент сплава со временем определяется уравнением непрерывности $[44,46]$ :

$$
\frac{d c}{d t}=-\nabla J, \quad J=-\frac{D(\mathbf{r}) c(1-c)}{k T} \nabla\left(\frac{\delta F}{\delta c}\right),
$$

где $F(c)$ - функционал Гинзбурга-Ландау

$$
f=\int\left[f(c, \mathbf{r})+\frac{\sigma R^{2}}{2}\left[(\nabla c)^{2}+(\nabla \eta)^{2}\right]\right] d \mathbf{r} .
$$

Здесь $\sigma$ - поверхностная энергия выделений, $R-$ параметр, определяющий ширину межфазной границы. Коэффициент диффузии $D(r)$ должен учитывать различие скоростей объемной диффузии и диффузии на дефекте

$$
D(\mathbf{r})=D_{b}+\left(D_{\mathrm{def}}-D_{b}\right) \Omega\left(\mathbf{r}-\mathbf{r}_{\mathrm{def}}\right) .
$$

Наконец, для эволюции параметра порядка $\eta$ используем релаксационное уравнение Аллена-Кана [47]

$$
\frac{d \eta}{d t}=-M(\mathbf{r}) \frac{\delta F}{\delta \eta}
$$

где $M(\mathbf{r})=\kappa_{0} D(\mathbf{r}) / k T-$ коэффициент, определяющий частоту скачков атомов между разными подрешетками, $\kappa_{0} \sim a^{-2}, a-$ параметр решетки $[31,44]$. Отметим, что используемый подход предполагает плавное изменение параметров порядка $c(\mathbf{r}), \eta(\mathbf{r})$ с координатой, что является определенным приближением, достаточным для демонстрации качественных особенностей рассматриваемых превращений.

При анализе решения системы (1)-(7) удобно использовать интегральные степени распада и упорядочения, $0<S_{\mathrm{dec}}<1,0<S_{\eta}<1$

$$
\begin{gathered}
S_{\mathrm{dec}}=\frac{1}{2 c_{0}\left(1-c_{0}\right) L^{n}} \int\left|c(\mathbf{r})-c_{0}\right| d \mathbf{r}, \\
S_{\eta}=\frac{2}{L^{n}} \int|\eta(\mathbf{r})| d \mathbf{r},
\end{gathered}
$$

где $n$ - размерность задачи, $c_{0}-$ средняя по образцу концентрация выбранного компонента. При численном интегрировании уравнений $(4)-(7)$ использовались безразмерные координаты $x / L$, время $\tau=\left(D_{\mathrm{def}} / L^{2}\right) t$ и скорость движения дефекта $v_{\mathrm{def}}=V_{\mathrm{def}} L / D_{\mathrm{def}}$.

Предложенная модель близка к модели регулярного твердого раствора [46], но отличается от нее учетом упорядочения (случай $\theta \neq 0$ ) и локальным изменением энергетических параметров на структурных дефектах (формулы (2)-(3)). В отличие от модели диффузионного перерезания выделений $[23,25]$ и от известных моделей сегрегаций на движущихся ГЗ $[39,41,42]$, данная модель учитывает изменение на дефекте не только энергии растворения примеси $\varepsilon(\mathbf{r})$, но также энергий смешения и упорядочения $v(\mathbf{r}), \theta(\mathbf{r})$, и не конкретизирует движущую силу миграции дефектов.

Параметризация модели обсуждается в Приложении.

\section{3. Сегрегации на движущихся границах зерен}

Формирование сегрегаций определяется изменением энергии примеси при перемещении ее из объема на границу зерна (энергией сегрегации) $\delta \varepsilon(r)[39,48]$. В условиях ИПД сегрегации могут возникать при достаточно низкой температуре $(\sim 300 \mathrm{~K})$, в широком приграничном слое (см. обсуждение в [26,49]). В работе [13] было высказано предположение, что кинетика формирования таких сегрегаций лимитируется не объемной диффузией, а процессами, протекающими на границе зерна. Движущаяся ГЗ увлекает примесные атомы, благодаря чему скорость формирования сегрегаций многократно возрастает.

Для демонстрации качественных особенностей взаимодействия движущихся дефектов с примесями, рассмотрим одномерную модель и ограничимся случаем идеального твердого раствора $(v=0, \theta=0)$ [46]. Сначала рассмотрим ситуацию, когда дефект движется с постоянной скоростью $V_{\text {def. }}$.

На рис. 1, $a$ приведено изменение со временем максимальной концентрации примеси на ГЗ, достигаемое при ее движении. Сегрегация на неподвижной ГЗ $\left(v_{\mathrm{GB}}=0\right)$ контролируется объемной диффузией $\left(D_{b} \ll D_{\mathrm{GB}}\right)$, и поэтому не успевает реализоваться на рассматриваемом масштабе времен (кривая 1). Напротив, движущаяся ГЗ увлекает примесные атомы, так что образование сегрегации определяется в основном скоростью ее движения (кривые 2-4). Если ГЗ движется с невысокой скоростью, примесные атомы успевают следовать за ней. Если скорость движения ГЗ выше критической, диффузия в направлении перпендикулярном границе не успевает обеспечить перераспределение атомов и концентрация на ГЗ падает (кривая 4). Приравнивая характерное диффузионное время, за которое атом пересекает границу $t=d^{2} / D_{\mathrm{GB}}$, ко времени за которое ГЗ смещается на расстояние равное своей ширине, $t=d / V_{\mathrm{GB}}$, получаем оценку оптимальной скорости движения ГЗ, при которой сегрегации развиваются наиболее эффективно:

$$
V_{\mathrm{GB}}^{(\mathrm{opt})}=D_{\mathrm{GB}} / d
$$

Эффект увлечения сегрегаций за движущейся ГЗ иллюстрируется на рис. $1, b$, где показана зависимость концентрации примеси на ГЗ, достигнутой к заданному моменту времени, от скорости движения ГЗ (кривые 1,2). 

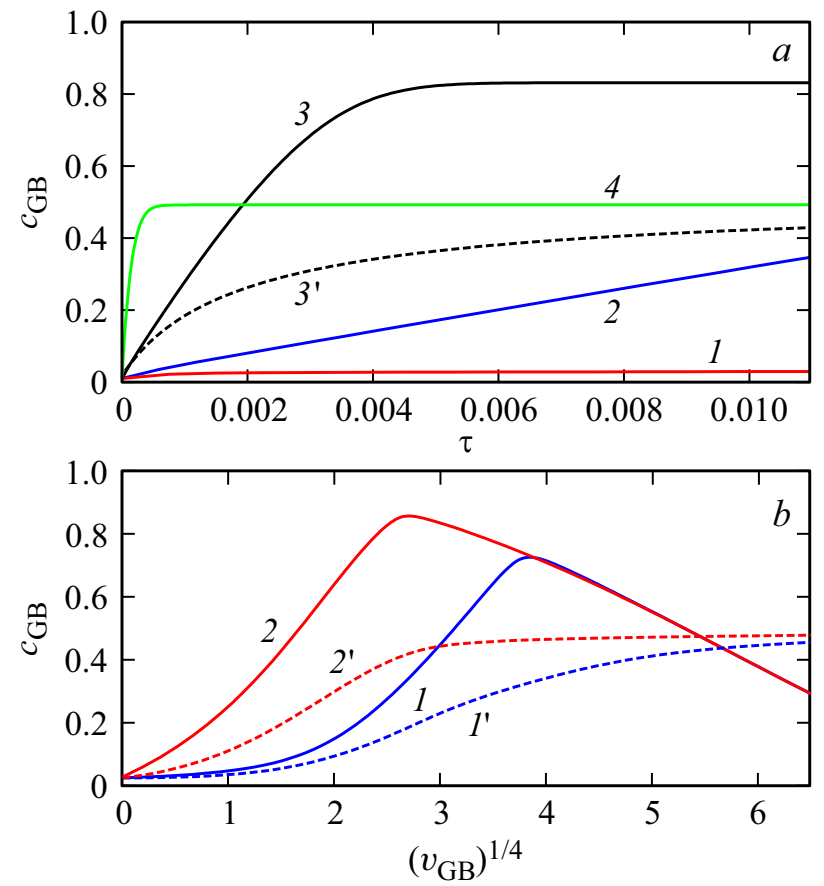

Рис. 1. $a-$ эволюция концентрации примеси на границе зерна при скоростях движения границы $v_{\mathrm{GB}}=0$ (1), 12.5 (2), 100 (3), 800 (4); кривая $3^{\prime}$ соответствует 3 с учетом торможения границы при $K=2 ; b-$ зависимость концентрации примеси на границе зерна, достигнутой к моменту времени $\tau=0.002\left(1,1^{\prime}\right), 0.02\left(2,2^{\prime}\right)$ от начальной скорости движения границы - в отсутствие $(1,2)$ и при наличии $\left(1^{\prime}, 2^{\prime}\right)$ торможения границы примесными атомами. Средняя концентрация примеси $c_{0}=0.01$, энергия сегрегации $\delta \varepsilon=-0.3 \mathrm{eV} / \mathrm{at}$, $T=500 \mathrm{~K}, D_{\mathrm{GB}} / D_{b}=10^{5}, d / L=0.005$.

Можно видеть, что с ростом времени максимальное значение $c_{\mathrm{GB}}$ возрастает и смещается в сторону меньших скоростей. Следовательно, в данной модели максимально возможная сегрегация ожидается на неподвижной ГЗ, но при бесконечно большой выдержке, что согласуется с результатами работы [37].

Согласно существующим представлениям [50], движение границы осуществляется миграцией уступов, которая контролируется самодиффузией атомов матрицы, либо реализуется атермически, благодаря действию механизмов зернограничного проскальзывания [51]. Наличие сегрегаций на ГЗ будет приводить к ее торможению благодаря взаимодействию примесных атомов с уступами, так что скорость движения ГЗ перестает быть независимым параметром. Учтем примесное торможение ГЗ в простейшей модели и представим скорость миграции в виде

$$
\tilde{v}_{\mathrm{GB}}(t)=v_{\mathrm{GB}}\left(1-K\left(c_{\max }(t)-c_{0}\right)\right)
$$

где $v_{\mathrm{GB}}, \tilde{v}_{\mathrm{GB}}(t)$ - соответственно начальная и текущая скорости $\Gamma 3, c_{\max }(t)$ - максимальная концентрация, достигаемая на ГЗ, $c_{0}-$ средняя (исходная) концентрация примеси в образце, $K$ - параметр, характеризующий эффективность торможения на примесях.

Кривая $3^{\prime}$ на рис. $1, a$, а также кривые $1^{\prime}, 2^{\prime}$ на рис. $1, b$ (описывающие зависимость концентрации на ГЗ от скорости движения границы $v_{\mathrm{GB}}$ ) показывают, что торможение ГЗ стабилизирует промежуточные стадии образования сегрегаций, вблизи критического значения $c_{\max }^{\mathrm{cr}}=c_{0}+1 / K$, при котором происходит „захват“ границы (скорость $\left.\tilde{v}_{\mathrm{GB}}(t) \equiv 0\right)$. Дальнейшее увеличение концентрации на ГЗ является медленным процессом, который лимитируется коэффициентом объемной диффузии $D_{b}$. Выход на насыщение кривых $1^{\prime}, 2^{\prime}$ на рис. $1, b$ выражает тот факт, что ГЗ останавливается при достижении сегрегации $c_{\max }^{\mathrm{cr}}$ в широком интервале значений начальных скоростей движения $v_{\mathrm{GB}}$.

Следует отметить, что максимальное значение концентрации, достигаемое на ГЗ (рис. 1), получено в пренебрежении взаимодействием между примесными атомами. При положительном значении энергии смешения $(v>0)$, отталкивание между примесными атомами будет ограничивать образование сегрегаций (смотри обсуждение в [26]), в то время как при $v<0$ следует ожидать образования выделений на ГЗ.

Аналогичное рассмотрение может быть проведено не только для сегрегаций на ГЗ, но также и при формировании примесных атмосфер на движущихся дислокациях. Однако скольжение дислокаций реализуется за времена много меньше характерных диффузионных времен. Поэтому ожидать появления рассмотренных эффектов следует только для относительно медленных механизмов миграции дислокаций (переползание, термоактивированное движение дислокаций в упорядоченных сплавах).

\section{4. Разупорядочение сплава и образование неравновесных фаз}

Изменение энергии упорядочения $\theta(\mathbf{r})$ вблизи дефекта (дислокации или ГЗ) приводит к локальному смещению температуры упорядочения. Действительно, из (1), (7) имеем условие Брэгга-Вильямса для локально-равновесного состояния $(d F / d \eta=0)$ [44]

$$
-\frac{4 \theta(\mathbf{r}) \eta(\mathbf{r})}{k T}=\ln \left[\frac{(c(\mathbf{r})+\eta(\mathbf{r}))(1-c(\mathbf{r})+\eta(\mathbf{r}))}{(c(\mathbf{r})-\eta(\mathbf{r}))(1-c(\mathbf{r})-\eta(\mathbf{r}))}\right] .
$$

Стартовая температура упорядочения возникает из (11) в пределе $\eta(\mathbf{r}) \rightarrow 0$

$$
k T_{\text {ord }}(\mathbf{r})=-2 \theta(\mathbf{r}) c(\mathbf{r})(1-c(\mathbf{r})) .
$$

Учитывая, что исходное упорядоченное состояние соответствует минимуму свободной энергии сплава (1), естественно ожидать, что в искаженной решетке вблизи дефекта свободная энергия упорядоченного состояния возрастает, т.е. $\delta \theta>0$. В результате, в соответствии 

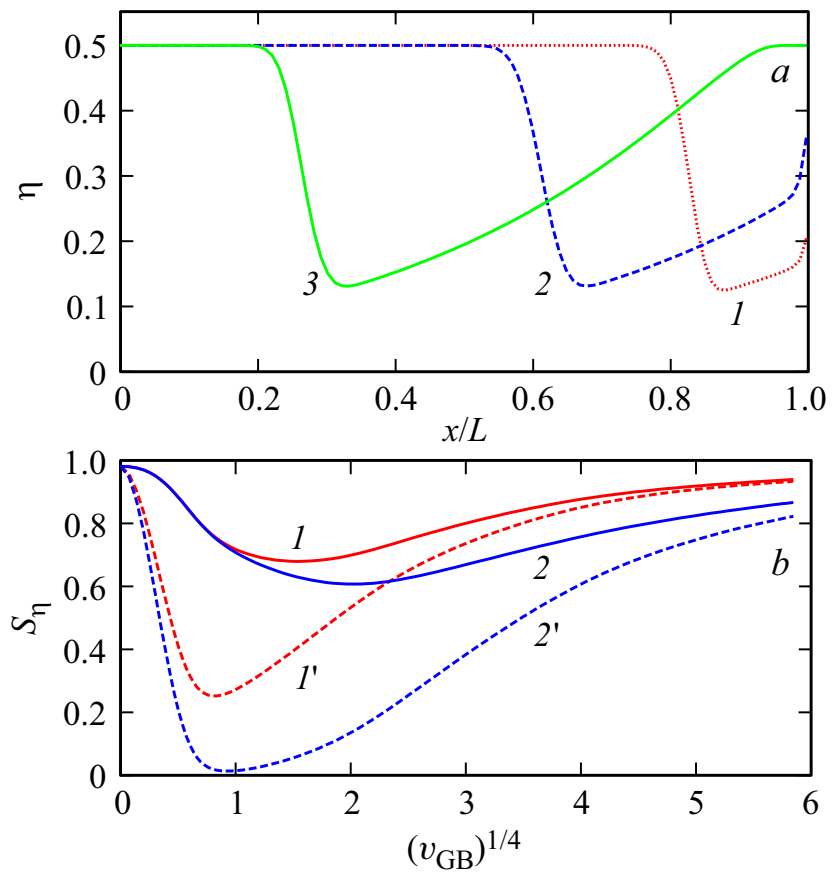

Pис. 2. $a-$ профили параметра порядка $\eta$ в моменты времени $\tau=0.02(1), 0.04$ (2), 0.07 (3) при смещении границы зерна из начального положения $x / L=1$ в положение с координатой $x / L=0$ со скоростью $v_{\mathrm{GB}}=10$ при $D_{\mathrm{GB}} / D_{b}=10^{2}$; $b-$ зависимость средней по образцу степени упорядочения от скорости движения дефекта после первого прохода $\left(1,1^{\prime}\right)$ и после пяти проходов $\left(2,2^{\prime}\right) ; D_{\mathrm{GB}} / D_{b}=10^{2}(1,2), 10^{5}\left(1^{\prime}, 2^{\prime}\right)$. $c_{0}=0.5, \kappa_{0} L^{2}=250, v=\delta \varepsilon=0, \theta_{b}=-0.3 \mathrm{eV}, \delta \theta=+0.3 \mathrm{eV}$, $T=500 \mathrm{~K}, d / L=0.03$.

с (12), следует ожидать понижения температуры упорядочения на дефекте,

$$
T_{\text {ord }} \rightarrow T_{\text {ord }}^{\text {def }}=T_{\text {ord }}-2 \delta \theta c(1-c) / k,
$$

а в температурном интервале $T_{\text {ord }}^{\text {def }}<T<T_{\text {ord }}$ будет наблюдаться локальное разупорядочение сплава. После смещении дефекта разупорядоченное состояние замораживается в силу различия коэффициентов диффузии $D_{\text {def }}, D_{b}$.

На рис. 2, $а$ приведены результаты расчетов локального параметра порядка $\eta$ в различные моменты времени при прохождении ГЗ. Видно, что позади движущейся ГЗ остается протяженная частично разупорядоченная область. Степень разупорядочения определяется скоростью движения ГЗ и интенсивностью протекания релаксационных процессов, то есть отношением $D_{\mathrm{GB}} / D_{b}$. Как видно из рис. $2, b$, существует некоторое значение скорости $v_{\mathrm{GB}}^{\mathrm{opt}}$ (значительно ниже скорости звука, см. Приложение), при которой достигается наибольшее нарушение атомного порядка; при $v_{\mathrm{GB}}<v_{\mathrm{GB}}^{\text {opt }}$ атомный порядок восстанавливается позади ГЗ, а при $v_{\mathrm{GB}}>v_{\mathrm{GB}}^{\mathrm{opt}}$ разупорядочение не успевает реализоваться. В случае повторяющихся проходов ГЗ через зерно (которые при моделировании реализованы с помощью периодических граничных условий) разупорядочение сплава продолжается (ср. кривые 1 и $2,1^{\prime}$ и $2^{\prime}$ ). Поэтому при большом отношении $D_{\mathrm{GB}} / D_{b}$ и длительном воздействии можно ожидать полного разупорядочения даже при низкой концентрации дефектов.

Рис. 3 показывает зависимость средней по образцу степени упорядочения от температуры в отсутствие ГЗ (кривая 1) и в стационарном режиме, достигаемом при многократном прохождении ГЗ через рассматриваемый объем (кривые $\left.2,2^{\prime}, 3,3^{\prime}\right)$. Можно видеть, что стартовая температура упорядочения $T_{\mathrm{ord}}^{\mathrm{def}}$ опускается тем ниже, чем выше отношение $D_{\mathrm{def}} / D_{b}$ или $\delta \theta /\left|\theta_{b}\right|$.

Выше мы полагали, что на дефекте изменяется только энергия упорядочения $(\delta \theta \neq 0, \delta \varepsilon=0)$. Если $\delta \varepsilon \neq 0$, развитие сегрегаций одного из компонент сплава может стимулировать его разупорядочение даже при $\delta \theta=0$. При смещении дефекта сегрегация увлекается вслед за ним, в то время как достигнутое разупорядоченное состояние может оказаться замороженным (см. рис. 4). С другой стороны, при торможении дефекта сегрегациями $(K \neq 0$, см. формулу $(10))$, разупорядочение

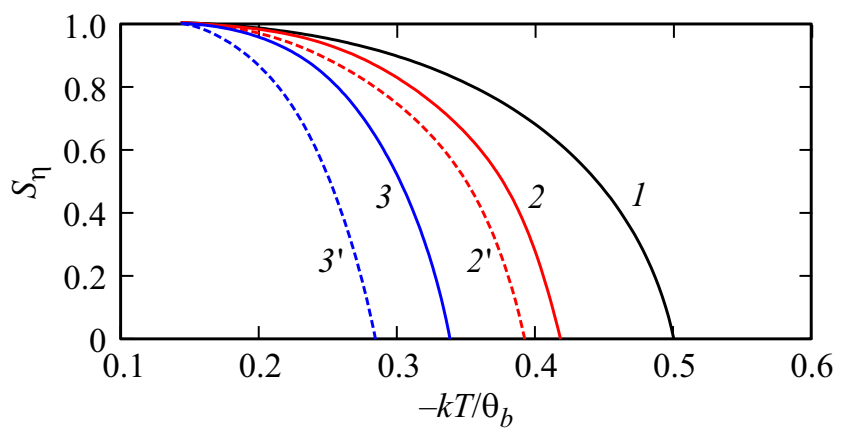

Рис. 3. Зависимость средней по образцу степени упорядочения от температуры: в отсутствие дефектов (1) и в стационарном режиме при многократном прохождении через образец ГЗ со скоростью $v_{\text {def }}=10 ; \delta \theta /\left|\theta_{b}\right|=0.25\left(2,2^{\prime}\right), 0.5\left(3,3^{\prime}\right)$, $D_{\mathrm{def}} / D_{b}=10^{2}(2,3), 10^{5}\left(2^{\prime}, 3^{\prime}\right)$. Остальные параметры такие же, как на рис. 2.

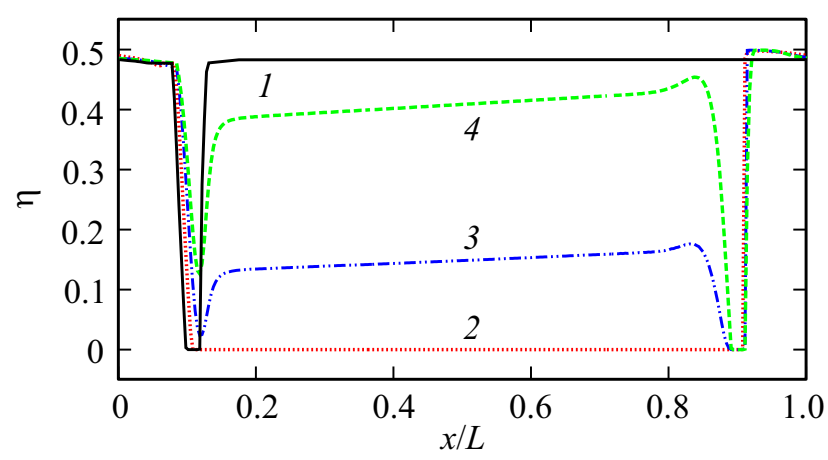

Рис. 4. Достигнутые профили параметра порядка $\eta$ после смещения ГЗ из позиции $x / L=0.9$ в позицию $x / L=0.1$ со скоростью $v_{\mathrm{GB}}=1 \quad(1), \quad 5 \quad$ (2), 15 (3), 25 (4); $c_{0}=0.5, \quad D_{\mathrm{GB}} / D_{b}=10^{3}, \quad \kappa_{0} L^{2}=500, \quad v=0, \quad \delta \varepsilon=-0.35 \mathrm{eV}$, $\theta_{b}=-0.3 \mathrm{eV}, T=500 \mathrm{~K}, d / L=0.05$. 

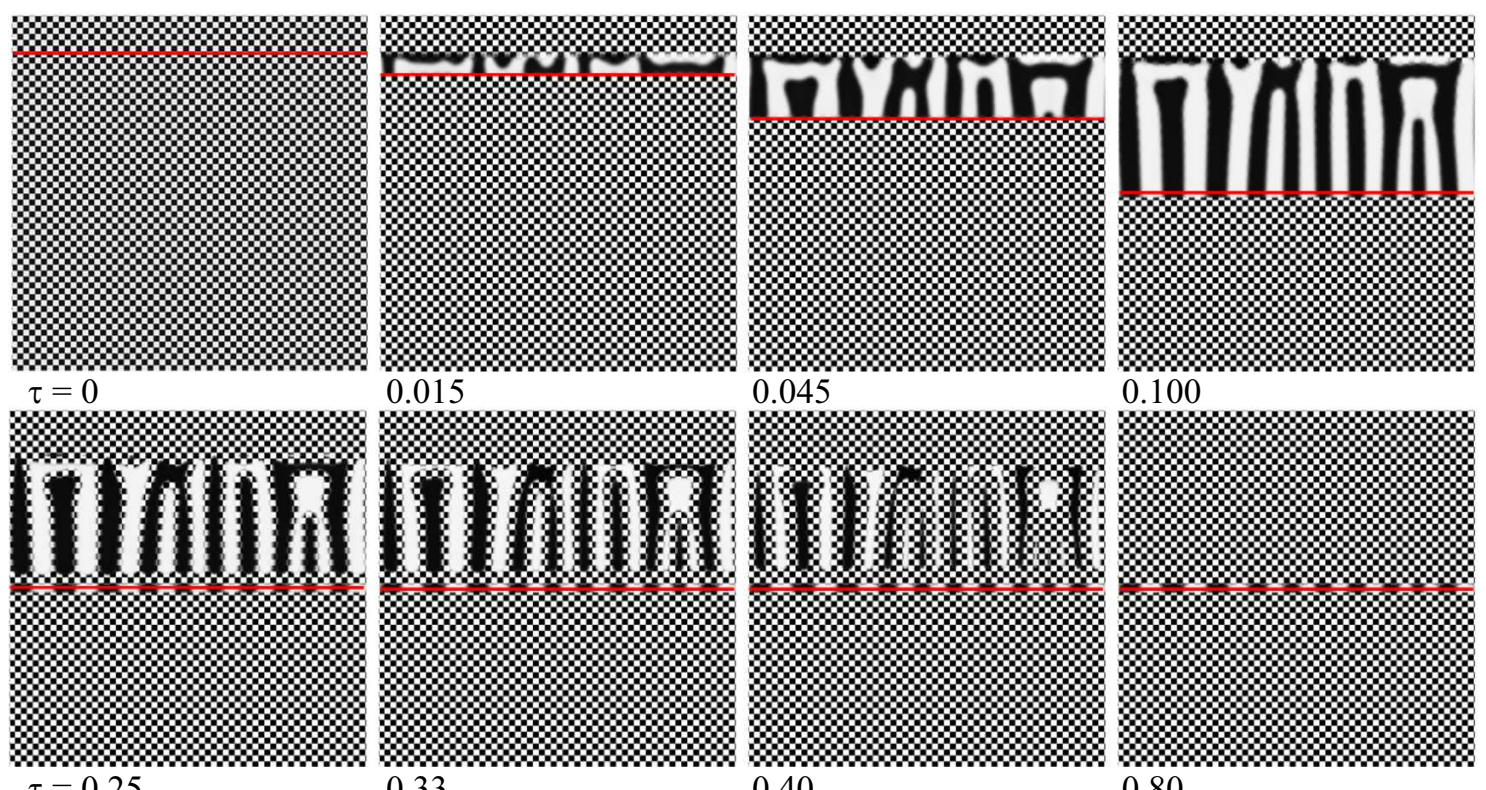
0.015 0.045

0.100
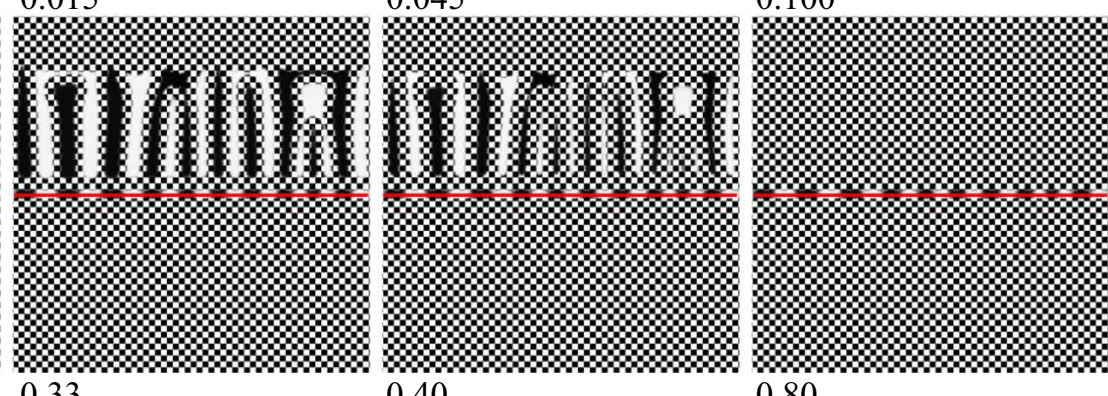

Рис. 5. Распад равновесной упорядоченной фазы, обусловленный изменением энергии упорядочения на ГЗ (ее положение отмечено горизонтальной линией), перемещающейся из позиции $y / L=0.9$ в позицию $y / L=0.5$, и восстановление упорядоченного состояния при выдержке; $\theta=-0.3 \mathrm{eV}, \delta \theta=+0.3 \mathrm{eV}, D_{\mathrm{GB}} / D_{b}=10^{2}, v_{\mathrm{GB}}=4, c_{0}=0.5, \kappa_{0} L^{2}=250, v=-0.14 \mathrm{eV}, \delta \varepsilon=0$, $T=500 \mathrm{~K}, d / L=0.03, R / L=0.0016$. Белым и черным цветом обозначены обедненная и обогащенная фазы, возникшие при распаде, остальная часть рисунка соответствует исходной упорядоченной фазе.

возможно только если начальная скорость дефекта выше критического значения, в противном случае сегрегации останавливают дефект и соответственно разупорядочение не реализуется.

В общем случае упорядоченное состояние в равновесии обеспечивается соотношением энергий $\theta<v$ (см. формулу (1)), то есть тенденция возможного распада подавлена за счет упорядочения. При этом, в области дефекта может произойти локальное разупорядочение в результате изменения энергии $\theta$, либо в результате сегрегации одного из компонент. Тогда на фоне разупорядоченного состояния возможно развитие спинодального распада.

На рис. 5 представлена кинетика распада упорядоченной фазы, когда энергия $\theta$ изменяется на границе зерна (ГЗ перемещается из позиции $y / L=0.9$ в позицию $y / L=0.5$ и останавливается). В процессе движения границы на ней развивается спинодальный распад (рис. 5, верхний ряд), а при последующей выдержке система релаксирует к равновесному упорядоченному состоянию со скоростью определяемой коэффициентом объемной диффузии $D_{b}$ (рис. 5 , нижний ряд).

\section{5. Растворение выделений и формирование диссипативных структур}

В отсутствие упорядочения $(\theta=0)$ при $v<0$ уравнение (4) описывает распад с образованием двухфазного состояния, причем равновесные пределы растворимости в фазах определяются бинодалью регулярного твердого раствора [46]

$$
\frac{v}{k T}=\frac{1}{1-2 c} \ln \left(\frac{c}{1-c}\right)
$$

Ограничимся рассмотрением ситуации, когда энергия смешения $v$ всюду одинакова, в то время как энергия растворения $\varepsilon(\mathbf{r})$ и коэффициент диффузии локально изменяются на дефекте (дислокации либо ГЗ), обеспечивая сегрегации одного из компонент. Если дефект неподвижен, сегрегации стимулируют появление выделений равновесных фаз, повышая степень распада. Однако, если дефект движется и пересекает объем выделений, может реализоваться другой сценарий, при котором происходит растворение выделений.

Пусть энергии сегрегации на дефекте $\delta \varepsilon$ в соседних фазах различаются, стимулируя перераспределение вещества вдоль дефекта между матрицей и объемом выделения. Если при этом энергия сегрегации в матрице больше (по абсолютной величине), чем в выделении, возникает поток атомов вдоль ГЗ (либо дислокационной трубки) из объема выделения в матрицу, способствующий растворению выделения. Термодинамический стимул распада, определяемый энергией смешения $v$, выступает при этом конкурирующим фактором.

Ограничимся рассмотрением простой модели и будем относить к выделению области, в которых $c(\mathbf{r})>0.5$. Тогда для энергии сегрегации примеси на дефекте 

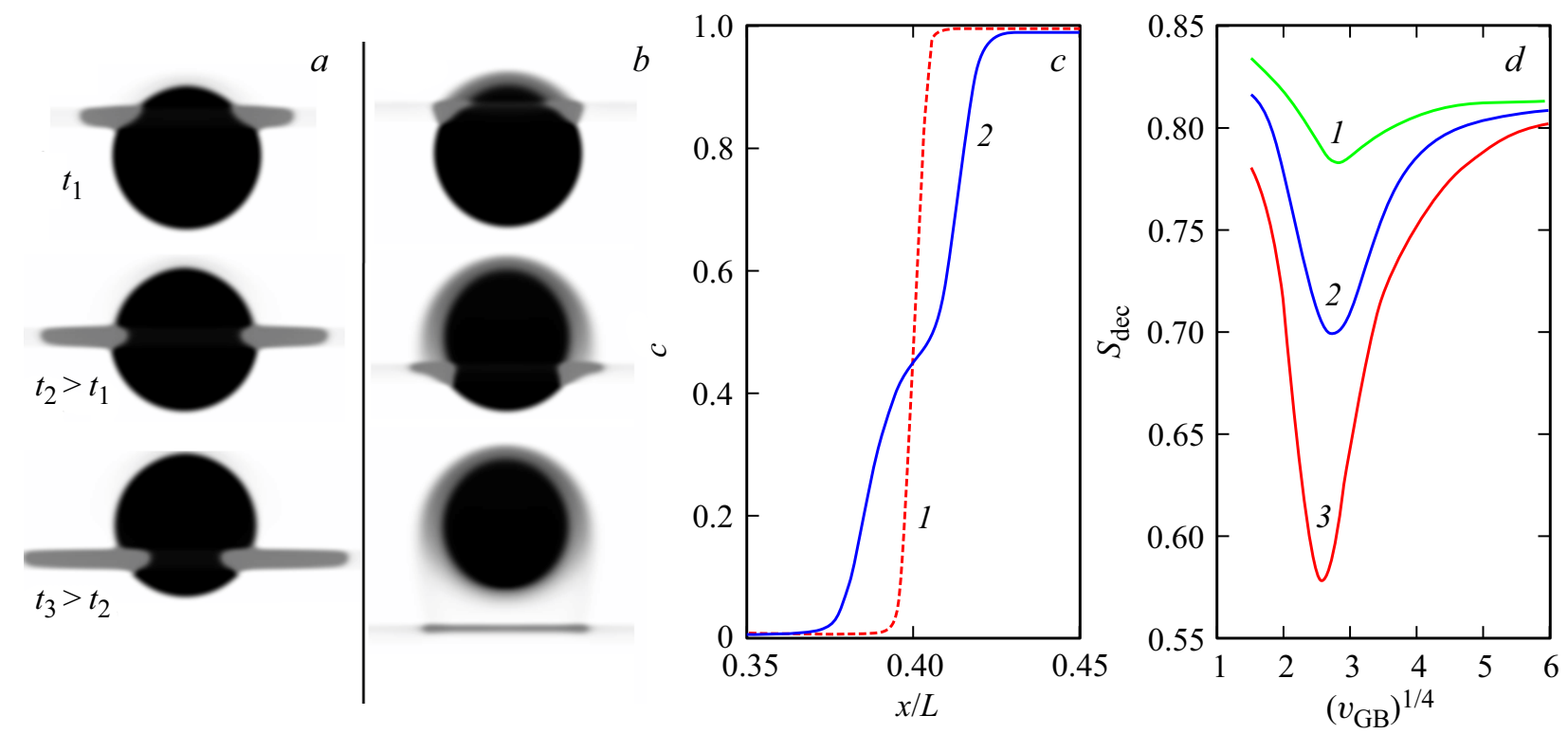

Рис. 6. Распределение концентрации компонента в окрестности выделения в различные моменты времени при однократном проходе ГЗ со скоростью $v_{\mathrm{GB}}=10(a), 60(b) ; c-$ профили концентрации компонента на интерфейсе исходного выделения (кривая 1) и после прохода Г3, $v_{\mathrm{GB}}=60$ (кривая 2); $d-$ зависимость степени распада от скорости движения ГЗ после одного (1), пяти (2) и пятнадцати (3) проходов. $D_{\mathrm{GB}} / D_{b}=10^{5}, \delta \varepsilon^{(m)}=-0.15, \delta \varepsilon^{(p)}=0.10, v=-0.3(\mathrm{eV} / \mathrm{at}), T=700 \mathrm{~K}$, $d / L=0.03, R / L=0.1$.

можно записать:

$$
\delta \varepsilon(\mathbf{r})=\delta \varepsilon^{(m)}+h[c(\mathbf{r})-0.5]\left(\delta \varepsilon^{(p)}-\delta \varepsilon^{(m)}\right),
$$

где $\delta \varepsilon^{(p)}, \delta \varepsilon^{(m)}$ - энергии сегрегации на участках границы в объеме выделения и за его пределами соответственно, $h(c)$ - сглаженная функция Хэвисайда (см. Приложение).

Подстановка (14) в формулу (1) приводит к результату, который можно трактовать как локальное изменение энергии смешения $v$ на величину $\delta \varepsilon^{(p m)}=\delta \varepsilon^{(p)}-\delta \varepsilon^{(m)}$. Тогда из (13) следует, что стартовая температура распада однородного сплава понижается на дефекте

$$
k T_{\mathrm{dec}}^{\mathrm{def}}=\left(v+\delta \varepsilon^{(p m)}\right) \frac{1-2 c_{0}}{\ln \left[c_{0} /\left(1-c_{0}\right)\right]}
$$

и при достаточно большой величине $\delta \varepsilon^{(p m)}$ возможно локальное растворение выделения.

На рис. 6, $a, b$ представлены результаты моделирования прохождения ГЗ через выделение равновесной фазы. Можно видеть, что при малой величине $v_{\mathrm{GB}}$ (рис. $6, a)$ образуется сегрегация промежуточного состава $(c \sim 0.5)$, которая полностью увлекается за дефектом, а форма выделения остается подобной исходному. При большой скорости ГЗ, когда $V_{\mathrm{GB}}>D_{\mathrm{GB}} / d$ (см. (9)), сегрегация не успевает следовать за ГЗ, в результате чего интерфейс выделения позади ГЗ аномально уширяется (рис. 6, $b$ ) и „замораживается““ в неравновесном состоянии в силу низкой скорости объемной диффузии. Профили концентрации на исходном (равновесном) интерфейсе и после прохождения ГЗ представлены на рис. 6,c. Таким образом, если при малых $v_{\mathrm{GB}}$ степень растворения выделения после однократного прохода ГЗ определяется сегрегационной емкостью ГЗ, то при больших $v_{\mathrm{GB}}$ площадью сечения выделений.

На рис. $6, d$ приведены зависимости средней по образцу степени распада $S_{\mathrm{dec}}$ от скорости движения ГЗ, при однократном проходе (кривая 1), после пяти (кривая 2) и пятнадцати (кривая 3) проходов. Можно видеть, что существует оптимальная скорость, при которой (для заданного числа проходов ГЗ) обеспечивается максимальная гомогенизация сплава.

Результат многократного прохождения дефектов через выделение зависит от соотношения энергий $\delta \varepsilon^{(p m)}$ и $v$, коэффициентов диффузии $D_{\text {def }}$ и $D_{b}$, от средней концентрации $c_{0}$ и от скорости движения дефектов. Многообразие возможных режимов сводится к трем основным сценариям: (1) выделение в целом сохраняется, но изменяется его размер (случай малого изменения $\left.\delta \varepsilon^{(p m)}\right)$, (2) выделение растворяется полностью, реализуется однородное состояние (случай низких температур и больших $\left.\delta \varepsilon^{(p m)}\right),(3)$ возникает дисперсное состояние (или модулированная структура), сохраняющее относительную стабильность в ходе дальнейшей деформации (случай умеренных температур).

На рис. 7 показаны типичные картины формирования модулированной структуры в процессе растворения одиночного выделения. Особенностью этого режима является относительно высокая скорость объемной диффузии $\left(D_{\mathrm{def}} / D_{b}=10^{2}\right)$, в результате чего возникает конкуренция между процессами, контролируемыми диффузией на дефектах и объемной диффузией, ответственной за возврат к двухфазному равновесию. Поэтому после прохода 


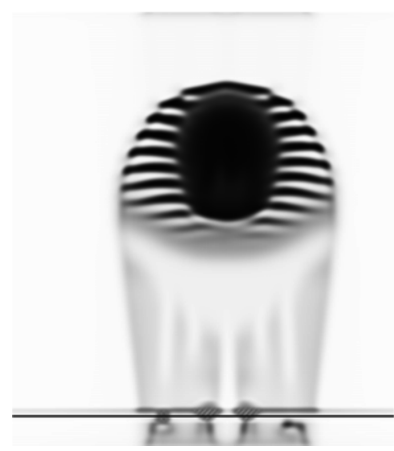

$\tau=0.30$

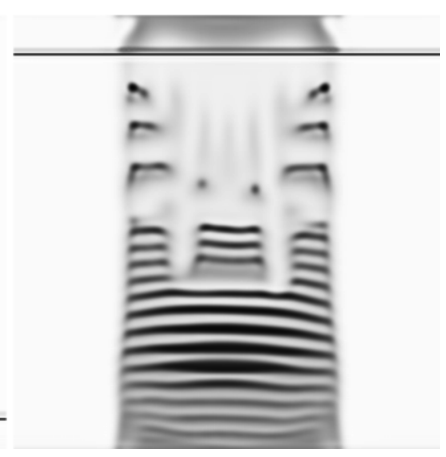

1.2
Рис. 7. Кинетика формирования модулированной структуры при многократном прохождении дефекта через выделение. $v_{\text {def }}=80, D_{\text {def }} / D_{b}=10^{2}, \delta \varepsilon^{(m)}=-0.15, \delta \varepsilon^{(p)}=+0.15$, $v=-0.3$ (eV/at), $T=700 \mathrm{~K}, d / L=0.03$.

дефекта в окрестности выделения развиваются процессы спинодального распада, скорость которых лимитируется объемной диффузией. Проход через выделение следующего дефекта приводит к уширению области с неравновесным составом. При больших временах воздействия исходное выделение полностью растворяется, и возникает квазипериодическое распределение концентраций, то есть происходит своего рода обращение спинодального распада.

\section{6. Обсуждение результатов}

Цель настоящей работы состояла в анализе качественных особенностей протекания неравновесных диффузионных превращений, инициированных движущимися дефектами при ИПД в области умеренных температур, когда объемная диффузия заморожена. Поэтому мы не рассматривали механический массоперенос при скольжении дислокаций, а ограничились простой моделью, учитывающей процессы аномальной диффузии на движущихся дефектах. Мы показываем, что локальное изменение параметров сплава в области протяженных структурных дефектов и ускоренная диффузия на них могут приводить к целому спектру неравновесных превращений. Полученные результаты дают возможность по-новому взглянуть на развитие структурных и фазовых превращений при ИПД в области умеренных температур.

В предложенной модели для большинства случаев не был конкретизирован тип дефектов (дислокации или границы зерен). Известно, что в крупнозеренных образцах преобладает дислокационная мода пластической деформации [20], а при переходе к нанокристаллическому (НК) состоянию активизируется низкотемпературная динамическая рекристаллизация, сопровождающаяся миграцией ГЗ [36]. Разупорядочение и растворение мелких выделений обычно не требуют перехода к НК состоянию [4,15], и в принципе могут обеспечиваться прохождением дислокаций. В то же время, для реализации ано- мального механосплавления в НК образцах требуется, по-видимому, миграция границ зерен. Отметим, что даже однократное прохождение ГЗ через образец способно привести к большим последствиям, сопоставимым с результатом прохода множества дислокаций.

В известных моделях формирования сегрегаций на движущихся Г3 [39] недостаточное внимание уделялось тому факту, что при низкой температуре кинетика сегрегаций контролируется зернограничной диффузией. Мы показали, что в этом случае движение ГЗ может быть критически важным для развития сегрегаций. При этом существует оптимальная скорость движения ГЗ, при которой сегрегации развиваются наиболее эффективно.

Разупорядочение интерметаллидов при ИПД было впервые обнаружено в системах $\mathrm{Fe}-\mathrm{Pt}, \mathrm{Co}-\mathrm{Pt}$, а в системах $\mathrm{Y}-\mathrm{Co}, \mathrm{Gd}-\mathrm{Co}$ оно сопровождалось аморфизацией [1]. Зачастую это превращение реализуется быстро, не требуя интенсивной и продолжительной обработки (см. обзоры $[2,17])$, что косвенно свидетельствует о невысокой плотности дефектов в объеме материала. Распространенное объяснение [17] этого явления прямым перемешиванием при скольжении неполных дислокаций через объем зерен - сталкивается с той трудностью, что по крайней мере в НК состоянии (которое зачастую предшествует разупорядочению и аморфизации) проникновение дислокаций в объем зерен подавлено [20]. Также разупорядочение сплава вряд ли может быть обеспечено движением сверхструктурных дислокаций, поскольку они относительно мало влияют на термодинамические параметры сплава и не нарушают химического порядка после своего прохождения. С другой стороны, необходимым условием перехода к НК состоянию называют низкотемпературную динамическую рекристаллизацию [36], что позволяет предполагать важную роль движущихся ГЗ в разупорядочении НК-сплавов.

Растворение выделений интерметаллидов и карбидов при ИПД неоднократно наблюдалось экспериментально (см. например [4]), причем для объяснения этого явления обычно привлекаются представления о механическом перемешивании в полосах скольжения [15-17], либо о „диффузионном перерезании“ [23-25], реализующемся при достаточно большом размерном несоответствии атомов матрицы и растворенного компонента (например, для примесей внедрения).

В предложенной нами модели рассматривается механическое перерезание выделений и учитывается, что кроме упругого, существенный вклад может давать изменение „химической“ энергии примесного атома. При проходе дефекта через выделение, в силу различия энергии сегрегации в выделении и матрице, возникают потоки атомов вдоль дефекта, что приводит к частичному растворению выделения и уширению межфазной границы (рис. 6,b). Последняя особенность является характерным признаком действия такого механизма. При многократном проходе дефектов возможно существенное измельчение и даже полное растворение выделе- 
ний. Подобный сценарий эволюции микроструктуры при ИПД наблюдался в $[4,52,53]$.

Изменение энергии химической связи на дефекте может приводить к тому, что если в одной фазе примесные атомы сегрегируют на ГЗ, то в другой фазе они, напротив, выталкиваются с ГЗ, то есть величина $\delta \varepsilon$ изменяет знак при переходе через межфазную границу, что и было реализовано в расчетах (рис. 6,7). Такое соотношение между энергиями сегрегации не является, строго говоря, обязательным. Для растворения выделений при их пересечении дислокациями или ГЗ достаточно, чтобы энергия примесного атома понижалась при перемещении из выделения в матрицу вдоль дефекта. Подобная ситуация реализуется, по-видимому, при пересечении частиц цементита дислокациями, сопровождающимся значительным обеднением цементита по углероду [53].

Примеры распада сплавов с образованием неравновесных фаз при ИПД обсуждались в [1,5-7,54-57]. Так, в частности, в системе $\mathrm{Nd}_{2} \mathrm{Fe}_{14} \mathrm{~B}$ происходило разупорядочение и аморфизация исходной фазы, а также выделение нанокристаллов $\alpha \mathrm{Fe}[5,6]$ (вопреки равновесной фазовой диаграмме). Представления о том, что реализация неравновесных превращений в этой системе связана с изменением термодинамических свойств сплава в результате накопленной энергии дефектов сформулированы в [56]. В рамках развиваемого подхода, рассматривающего движущиеся дефекты, предположение о их накоплении в материале не является обязательным. Следует отметить, что в этих экспериментах процессы диффузии реализуются вероятно лишь локально, в области полос сдвига; в противном случае объемная диффузия способствовала бы восстановлению атомного порядка и релаксации аморфного состояния.

В условиях ИПД при умеренных температурах наблюдалось также формирование стационарных дисперсных состояний (модулированных структур) в сплавах $\mathrm{AgCu}[8]$ и $\mathrm{FeCr}[9]$, обусловленных конкуренцией объемной диффузии (которая обеспечивает распад в согласии с равновесной фазовой диаграммой), и развития неравновесного превращения (механосплавления) в ходе воздействия. По-видимому, эти состояния могут рассматриваться как пример диссипативных структур [58], которые возникают в открытых системах, вдали от термодинамического равновесия. Согласно представлениям $[8,17]$ механосплавление обеспечивается измельчением выделений и прямым перемешиванием атомов в результате скольжения дислокаций в пересекающихся плоскостях. Однако, согласно [3], механосплавление ускоряется при переходе к НК состоянию, когда проникновение дислокаций в объем зерен затруднено [20], что, по-видимому, указывает на важную роль миграции ГЗ в этом случае. Наша модель выявляет новый механизм механосплавления и формирования диссипативных структур, связанный с диффузией на движущихся дефектах (дислокациях и границах зерен), который может реализоваться при умеренных температурах, когда эта диффузия не является пренебрежимо малой.
Обсуждаемые результаты получены в рамках простой модели, не учитывающей деталей взаимодействия примесных атомов с дислокациями или ГЗ и в предположении о постоянной скорости движения дефектов. Тем не менее, можно полагать, что сделанные выводы правильно отражают качественные особенности изменения фазового и структурного состояния сплавов при ИПД.

\section{7. Выводы}

Сформулирована модель, описывающая влияние движущихся дефектов (дислокации, границы зерен) на развитие неравновесных фазовых превращений при интенсивной пластической деформации. Показано, что процессы разупорядочения сплава, реализации неравновесных фаз, формирования пересыщенных твердых растворов и диссипативных структур могут реализоваться за счет локального изменения термодинамических свойств сплава и ускоренной диффузии на движущихся дефектах. Показано, что при однократном проходе дефекта существует оптимальная скорость его движения, при которой указанные превращения реализуются наиболее эффективно, а при многократных проходах имеет место накопительный эффект. Полученные результаты позволяют дать качественное объяснение особенностей изменения фазового и структурного состояния сплавов при ИПД на умеренных температурах, когда диффузия на дефектах не является пренебрежимо малой.

\section{Приложение. Параметризация модели}

В представленных расчетах размер зерна определяется отношением $L / d$, и составляет $30-200 \mathrm{~nm}$ (характерная ширина дефекта $d$ принята равной $1 \mathrm{~nm}$ ). Функция формы возмущения вблизи дефекта принималась в виде:

$$
\Omega\left(\mathbf{r}-\mathbf{r}_{\mathrm{def}}\right)=\left(1+\left[\frac{2}{d}\left(\mathbf{r}-\mathbf{r}_{\mathrm{def}}\right)\right]^{12}\right)^{-1} .
$$

При переключении энергии растворения в фазах, в зависимости от концентрации (см. формулу (14)), использовалась сглаженная функция Хэвисайда:

$$
h=\left(1+\exp \left(-\frac{c(\mathbf{r})-0.5}{0.02}\right)\right)^{-1} .
$$

Температура выбиралась из интервала $500-700 \mathrm{~K}$, типичные значения $D_{\mathrm{GB}}$ при этих температурах составляют $10^{-13}-10^{-19} \mathrm{~m}^{2} / \mathrm{s}$ [59]. Исходя из формулы $v_{\text {def }}=V_{\text {def }} L / D_{\text {def }}$ рассматриваемый в расчетах диапазон скоростей движения дефектов $V_{\text {def }}$ составляет $10^{-5}-10^{-14} \mathrm{~m} / \mathrm{s}$, а характерные времена процессов, исходя из формулы $\tau=D_{\operatorname{def}} / L^{2} t$ оцениваются с разбросом значений $10^{-4}-10^{+5} \mathrm{~s}$. В экспериментах аномальные превращения при ИПД протекают, как правило, за несколько секунд, что в принципе согласуется с этой оценкой. Следует обратить внимание, что генерация 
неравновесных вакансий в условиях ИПД [60] может повысить коэффициент диффузии $D_{\text {def }}$ (и соответственно характерные скорости процессов) на 10 порядков. Кроме того, коэффициент зернограничной диффузии на неравновесных ГЗ, которые формируются при ИПД, может возрастать на 3-5 порядков по сравнению с обычными условиями, в результате искажения решетки в широкой приграничной области [61].

Скорость процессов упорядочения в теоретических моделях $[44,45]$ определяется временем одного межузельного обмена, что много меньше характерных диффузионных времен, откуда следует $\kappa_{0} L^{2} \gg 1$. В экспериментах наблюдается инкубационный период упорядочения $[11,62]$, обусловленный вероятно необходимостью реализации дальнего порядка и, в определенных случаях, перестройкой кристаллической решетки, замедляющий развитие этого превращения.

\section{Список литературы}

[1] А.Е.Ермаков. ФММ 11, 4 (1991).

[2] H. Bakker, P.I. Loeff, A.W. Weeber. Def. Diff. Forum 66-69, 1169 (1989).

[3] C. Suryanarayana. Prog. Mater. Sci. 46, 1 (2001).

[4] В.В. Сагарадзе, В.А. Шабашов. ФММ 112, 2, 155 (2011).

[5] А.Г. Попов, В.С. Гавико, Н.Н. Щеголева. ФММ 104, 3, 238 (2007).

[6] А.Г. Попов, В.С. Гавико, А.С. Ермоленко, Н.Н. Щеголева, В.В. Столяров, Д.В.Гундеров. В сб.: Структура и свойства нанокристаллических материалов. Екатеринбург (1999). 245 c.

[7] В.А. Цурин, В.А. Баринов, С.Б. Пупышев. Письма в ЖТФ 21, 12, 20 (1995).

[8] F. Wu, D. Isheim, P. Bellon, D.N. Seidman. Acta Mater. 54, 2605 (2006).

[9] G. Le Caër, S. Begin-Colin, P.Delcroix. In: Materials Research in Atomic Scale by Mossbauer Spectroscopy / Ed. M. Mashlan, M. Miglierini, P. Schaaf (2003). P. 11-20.

[10] B.B. Straumal, S.G. Protasova, A.A. Mazilkin, E. Rabkin, D. Goll, G. Schutz, B. Baretzky, R.Z. Valiev. J. Mater. Sci. 47, 360 (2012).

[11] О.С. Новикова, А.Ю. Волков. ФММ 114, 2, 179 (2013).

[12] M. Herbig, D. Raabe, Y.J. Li, P.Choi, S. Zaefferer, S. Goto. Phys. Rev. Lett. 112, 12, 126103 (2014).

[13] B.B. Straumal, B. Baretzky, A.A. Mazilkin, F. Philipp, O.A. Kogtenkova, M.N. Volkov, R.Z. Valiev. Acta Mater. 52, 4469 (2004).

[14] X. Sauvage, A. Ganeev, Y. Ivanisenko, N. Enikeev, M. Murashkin, R. Valiev. Adv. Eng. Mater. 14, 11, 968 (2012).

[15] H. Bakker, G.F. Zhou, H. Yang. Prog. Mater. Sci. 39, 3, 159 (1995).

[16] P. Bellon, R. Averback. Phys. Rev. Lett. 74, 1819 (1995).

[17] G. Martin, P. Bellon. Solid State Phys. 50, 189 (1997).

[18] Г.А. Дорофеев, Е.П. Елсуков. ФММ 103, 6, 626 (2007).

[19] E.P. Yelsukov, G.A. Dorofeev, V.A. Barinov, T.F. Grigor'eva, V.V. Boldyrev. Mater. Sci. Forum 269-272, 151 (1998).

[20] В.В. Рыбин. Большие пластические деформации и разрушение металлов. Металлургия, М. (1986). 224 с.
[21] J. Eckert, J. Holzer, C. Krill, W. Johnson. J. Appl. Phys. 73, 2794 (1993).

[22] М.А. Штремель. МиТОМ, 8, 10 (2002).

[23] H. Gleiter. Acta Meter. 16, 455 (1968).

[24] Б.Я. Любов, В.А. Шмаков. ФММ 29, 5, 968 (1970).

[25] Б.Я. Любов. Диффузионные процессы в неоднородных твердых средах. Наука, М. (1981). 296 с.

[26] L.E. Karkina, I.N. Karkin, A.R. Kuznetsov, I.K. Razumov, P.A. Korzhavyi, Yu.N. Gornostyrev. Comp. Mater. Sci. 112, 18 (2016).

[27] A.Y. Lozovoi, A.T. Paxton, M.W. Finnis. Phys. Rev. B 74, 155416 (2006).

[28] Р. Хоникомб. Пластическая деформация металлов. Мир, M. (1972) $408 \mathrm{c}$.

[29] B.Q. Li, F.E. Wanter. Acta Mater. 46, 5483 (1998).

[30] A.R. Yavari, P.J. Desré, T. Benameur. Phys. Rev. Lett. 68, 2235 (1992).

[31] И.К. Разумов. ЖФХ 84, 9, 1632 (2010).

[32] R.D. Doherty. Met. Sci. 16, 1, 1 (1982).

[33] H. Octor, S. Naka. Phil. Mag. Lett. 59, 5, 229 (1989).

[34] S. Naka, H. Octor, E. Bouchaud, T. Khan. Scripta Met. 23, 4, 501 (1989).

[35] E. Bouchaud, S. Naka, H. Octor. Colloque Phys. 51, 451 (1990).

[36] А.М. Глезер, М.С. Метлов. ФТТ 52, 6, 1090 (2010).

[37] J.W. Cahn. Acta Met. 10, 9, 789 (1962).

[38] K. Lücke, H.P. Stüwe. Acta Met. 19, 1087 (1971).

[39] P. Lejček. Grain boundary segregation in metals. Springer Series in Materials Science, Berlin (2010). 250 p.

[40] G. Gottstein, L.S. Shvindlerman. Grain Boundary Migration in Metals: Thermodynamics, Kinetics, Applications. CRC Press, Boca Raton (1999).

[41] P.R. Cha, S.G. Kim, D.H. Yeon, J.K. Yoon. Acta Mater. 50, 3817 (2002).

[42] J. Li, J. Wang, G. Yang. Acta Mater. 57, 2108 (2009).

[43] А.Г. Хачатурян. Теория фазовых превращений и структура твердых растворов. Наука, М. (1974), 384 с.

[44] J.-F. Gouyet, M. Plapp, W. Dieterich, P. Maas. Adv. Phys. 52, 6, 523 (2003).

[45] I.K. Razumov, Yu.N. Gornostyrev, A.Ye. Yermakov. Rev. Adv. Mater. Sci. 18, 767 (2008).

[46] Дж. Кристиан. Теория превращений в металлах и сплавах. Мир, М. (1978). 806 c.

[47] S.M. Allen, J.W. Cahn. J. Phys. 38, 7 (1977).

[48] Д. МакЛин. Границы зерен в металлах. Металлургиздат, М. (1960). 332 c.

[49] И.К. Разумов. ЖФХ 88, 3, 485 (2014).

[50] G. Gottstein, D.A. Molodov, L.S. Shvindlerman. Interface Sci. 6, 7 (1998).

[51] D.S. Gianola, S. Van Petegem, M. Legros, S. Brandstetter, H. Van Swygenhoven, K.J. Hemker. Acta Mater. 54, 2253 (2006).

[52] В.Т. Ракин, Н.Н.Буйнов. ФММ 11, 59 (1961).

[53] Yu. Ivanisenko, W. Lojkowski, R.Z. Valiev, H.-J. Fecht. Acta Mater. 51, 5555 (2003).

[54] V.A. Barinov, G.A. Dorofeev, L.V. Ovechkin, E.P. Elsukov, A.E. Ermakov. Phys. Status Solidi A 123, 2, 527 (1991).

[55] A.Ye. Yermakov. Mater. Sci. Forum 179-181, 455 ( 1995).

[56] Е.И. Тейтель, Л.С. Метлов, Д.В. Гундеров, А.В. Корзников. ФММ 113, 1230 (2012). 
[57] И.К. Разумов, Ю.Н. Горностырев, А.Е. Ермаков. ФММ 119, 12 (2018). В печати.

[58] И. Пригожин. Введение в термодинамику необратимых процессов. НИЦ „Регулярная и хаотическая динамика“, Ижевск (2001). 160 с.

[59] Diffusion in Solids Metals and Alloys, Landolt-Börnstein New Series V. III/26. Springer-Verlag, Berlin (1990).

[60] T. Ungar, E. Schafler, P. Hanak, S. Bernstorff, M. Zehetbauer. Mater. Sci. Eng. A 462, 398 (2007).

[61] В.В. Попов, А.В. Сергеев. ФММ 118, 11, 1149 (2017).

[62] B. Gwalani, T. Alam, C.Miller, T. Rojhirunsakool, Y.S. Kim, S.S. Kim, M.J. Kaufman, Yang Ren, R. Banerjee. Acta Mater. 115, 372 (2016).

Редактор Ю.Э. Китаев 